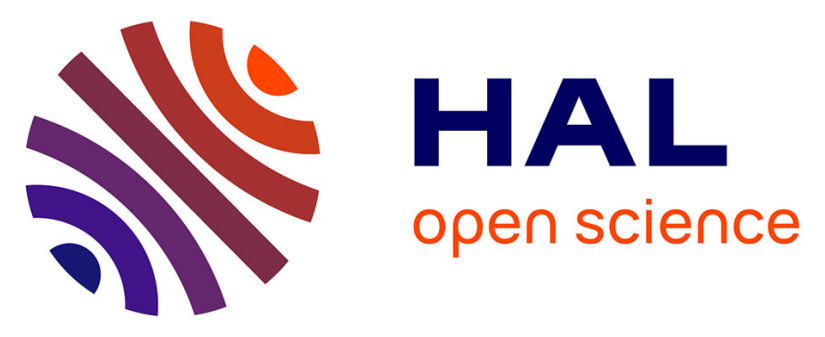

\title{
The Impacts of Five Different Turbulence Models on the Accuracy of Computational Aeroacoustic Results for an Airfoil and Acoustic Localization Analysis for Well Suited Model
}

\author{
Daniel Saatchi, Mani Fathali, Ali Rahimi Khojasteh
}

\section{To cite this version:}

Daniel Saatchi, Mani Fathali, Ali Rahimi Khojasteh. The Impacts of Five Different Turbulence Models on the Accuracy of Computational Aeroacoustic Results for an Airfoil and Acoustic Localization Analysis for Well Suited Model. International Review of Mechanical Engineering (I.RE.M.E.), 2014, 8 (2), pp.387. 10.15866/ireme.v8i2.1393 . hal-03328629

\section{HAL Id: hal-03328629 \\ https://hal.inrae.fr/hal-03328629}

Submitted on 30 Aug 2021

HAL is a multi-disciplinary open access archive for the deposit and dissemination of scientific research documents, whether they are published or not. The documents may come from teaching and research institutions in France or abroad, or from public or private research centers.
L'archive ouverte pluridisciplinaire HAL, est destinée au dépôt et à la diffusion de documents scientifiques de niveau recherche, publiés ou non, émanant des établissements d'enseignement et de recherche français ou étrangers, des laboratoires publics ou privés. 


\title{
The Impacts of Five Different Turbulence Models on the Accuracy of Computational Aeroacoustic Results for an Airfoil and Acoustic Localization Analysis for Well Suited Model
}

\author{
D. Saatchi ${ }^{1}$, M. Fathali ${ }^{2}$, A. R. Khojasteh ${ }^{3}$
}

\begin{abstract}
The research consists of two main sections; it initially focused on the accuracy of turbulence models to simulate the acoustic far-field domain for a NACA 0012 airfoil at Mach number of 0.209. Five different turbulence models were used such as realizable $k-\varepsilon$, SST $k-\omega$, Transition-SST, Reynolds-Stress, and Spalart-Allmaras in unsteady flow at velocity of $71.3 \mathrm{~m} / \mathrm{s}$. It used the Ffowcs-William and Hawkings (FW-H) acoustic method to compare the one-third-octaveband of semi-logarithmic sound pressure level (SPL) vs. frequency within the range of $200 \mathrm{~Hz}$ to $20000 \mathrm{~Hz}$ with experimental data. Reasonably good agreement is observed that both realizable $k$ $\varepsilon$ and Transition-SST are more accurate than others in prediction of peak of sound pressure level and the frequency of peak. In second section, in retrospect to considering accuracy of realizable $k$ $\varepsilon$ model and its shorter runtime, the paper considered some analysis on the acoustic far-field domain to find the critical points of fluctuated pressure due to the retard time. For this reason, a need to find a way to input the location of receiver to compare its data with other located receivers was felt. Indeed, it defined two different major distributions, semi-circle and far-field linear distribution, that each one has nineteen receivers. These distributions have mathematical correlations derived in their own subsection. It also introduces a parameter, Average-Dependable $S P L$, in order to analyze the effect of location and retard time on the fluctuations. After specification of six critical points in schematic contours, it is developed to a third distribution that corresponds to the proportion of chord. Finally, it can be observed that majority of peaks occur close to the turbulent boundary layer of trailing edge, but the quantity of peaks near the leading edge is more substantial than trailing edge peaks. Copyright (C) 2014 Praise Worthy Prize S.r.l. All rights reserved.
\end{abstract}

Keywords: Acoustic, Turbulence, Airfoil, SPL, Frequency, Fluctuated Pressure

\section{Nomenclature}

Specific Reynolds stress tensor

Dissipation per unit mass

Favre-average temperature

Total energy

Total enthalpy

Favre-Average specific internal energy

Specific enthalpy

Kinetic energy

Molecular viscosity

Eddy viscosity

Dirac delta function

Heaviside function

Unit normal vector embedded to exterior

Time differentiation of $M_{r}$

Local source Mach number vector

Compressive stress tensor $p^{\prime} \quad$ Sound pressure at far-field

$p_{T}^{\prime}(\vec{x}, t) \quad$ Thickness term

$p_{L}^{\prime}(\vec{x}, t) \quad$ Loading term

$P P E \quad$ Predicted peak error

$P F P E \quad$ Predicted frequency of peak error

$\bar{R} \quad$ Perfect gas constant

$R \quad$ Radius of semi-circle distribution

$R^{\prime} \quad$ Radius of far-field linear distribution

$r \quad$ Distance between receiver and source

$\vec{r} \quad$ Unit vector in the $i$ direction

$k \quad$ Kinetic energy of turbulent fluctuations

$\varepsilon \quad$ Dissipation per unit mass

$\omega \quad$ Specific dissipation rate

$\tau \quad$ Retard time

$\rho \quad$ Flow density

$\rho_{0} \quad$ Far-field density

$\delta_{i j} \quad$ Kronecker delta

$\theta \quad$ Angle of semi-circle distribution

$\theta^{\prime} \quad$ Angle of far-field linear distribution 


$\begin{array}{ll}C_{0} & \text { Local sound speed } \\ q_{T_{j}} & \text { Turbulent mean heat-flux vector } \\ q_{L_{j}} & \text { Laminar mean heat-flux vector } \\ v & \text { Kinematic molecular viscosity } \\ I_{D} & \text { Average-dependable SPL } \\ t_{j i} & \text { Instantaneous velocity in tensor notation } \\ t & \text { Time } \\ L E & \text { Leading edge } \\ L_{r} & \vec{L} \cdot \vec{r} \\ L_{M} & \vec{L} \cdot \vec{M}=L_{i} \cdot M_{i} \\ T_{i j} & \text { Lighthill stress tensor } \\ T R E & \text { Total residual error } \\ T B L & \text { Turbulent boundary layer } \\ T E & \text { Trailing edge } \\ v_{n} & \text { Surface velocity normal to surface } \\ v_{i} & \text { Surface velocity in the } x_{i} \text { direction } \\ u_{n} & \text { Fluid velocity normal to surface } \\ u_{i} & \text { Fluid velocity in the } x_{i} \text { direction } \\ \tilde{u}_{i} & \text { Favre-Average velocity in tensor notation } \\ u_{i}^{\prime \prime} & \text { Favre fluctuation velocity in tensor notation } \\ U_{i} & \text { Mean velocity in tensor notation } \\ U_{n} & \vec{U} \cdot \vec{n} \\ \dot{U}_{n} & \overrightarrow{\dot{U}} \cdot \vec{n} \\ S P L & \text { Sound pressure level } \\ S P L_{1 / 3} & \text { One-third of octave band SPL } \\ S_{i j} & \text { Mean strain-rate tensor } \\ \end{array}$

\section{Introduction}

Noise production is important for many engineering applications, such as helicopter-blade-noise, wind turbine, automotive and aerospace industries [1]-[21].

Environmental pressures to decrease fuel burn, emission and noise continue to drive the need for quieter, more efficient automobile, and aircraft propulsion technology. These environmental challenges also offer an opportunity for mechanical engineers and aircraft designers to take advantage of synergistic interaction between components of configuration design. Turbulent boundary layer of trailing edge noise is considered to be a major noise source in many aerodynamic applications, which is problematic [1].

The development of revolutionary technology improves the issue of how to optimize and integrate it. Aeroacoustics engineers seek to reduce sound intensity and sound pressure level to overcome noise production as well as Air Pollution engineers who attend to the level of substance and quality of air. Airfoil self-noise is due to the interaction between an airfoil blade and the turbulence produced in its own boundary layer and near wake. It is the total noise produced when an airfoil encounters smooth nonturbulent inflow.
Previous research effort (Prior to 1983) for the broadband noise mechanisms are reviewed in some detail by Brooks and Schlinker [2].

In Fig. 1, the subsonic flow conditions for self-noise mechanisms of concern here are illustrated. At high Reynolds number based on chord length, turbulent boundary layers (TBL) develop over most of the airfoil. Noise is produced as this turbulent passes over the trailing edge (TE).

For nonzero angle of attack, the flow can separate near the TE on the suction side of the airfoil to produce TE noise due to the shed turbulent vorticity Fig. 2. Another noise source is vortex shedding occurring in the small separated flow region aft of a blunt TE Fig. 3.

The remaining source considered here is due to the formation of the tip vortex, containing highly turbulent flow, occurring near the tips of lifting blades or wings Fig. 4 [3].

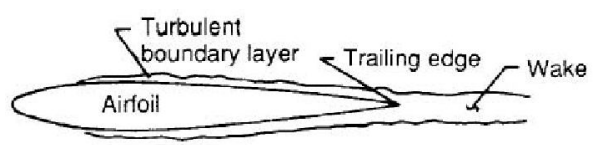

Fig. 1. Turbulent-boundary-layer-trailing-edge noise [3]

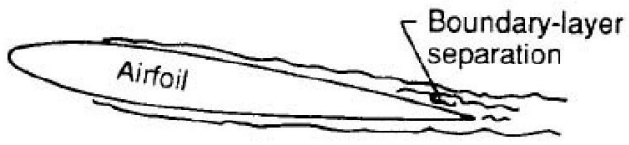

Fig. 2. Separation noise [3]

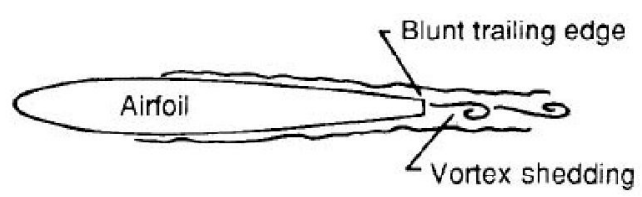

Fig. 3. Trailing-edge-bluntness-vortex-shedding noise [3]

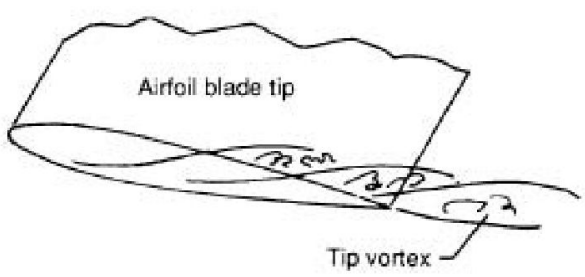

Fig. 4. Tip vortex formation noise [3]

The research kernel is based on the accuracy of five different turbulence models such as realizable $k-\varepsilon$, SST $k$ $\omega$, Transition-SST, Reynolds-Stress, and SparatAllmaras to plot the far-field acoustic semi-logarithmic Sound Pressure Level (SPL) vs. Frequency curve.

It concerns the acoustic criteria to compare each of turbulence models with brooks' experimental data [3].

Table I shows the subsonic flow specification for a NACA 0012 Airfoil to compute the numerical simulation of Reynolds-Average-Navier-Stokes (RANS) equations. 
In addition, Computational Aeroacoustics (CAA) for far-field acoustic domain due to the un-tripped TBL concerns Ffowcs-William Hawkings (FW-H) method. Subsection II-1 illustrates the fluid equations and subsection II-2 illustrates the noise prediction method.

In section VI, this paper will considers an analysis of acoustic localization for that turbulence model having shorter runtime in comparison with other ones.

Finally, the computations produced the results to select the appropriate turbulence models to do further research in near future.

TABLE I

FLOW SPECIFICATION

\begin{tabular}{rrrrr}
\hline \hline $\mathrm{U}(\mathrm{m} / \mathrm{s})$ & Mach & Reynolds & $\mathrm{c}(\mathrm{cm})$ & AoA \\
\hline 71.3 & 0.209 & 1108761 & 22.86 & zero \\
\hline \hline
\end{tabular}

\section{Governing Equations}

Governing equations are divided in Favre-equations and FW-H equations that need to cite initially.

\section{II.1. Compressible RANS Equations}

The RANS equations are time-averaged equations of motion for fluid flow. The idea behind the equations is Reynolds decomposition, whereby an instantaneous quantity is decomposed into its time-averaged and fluctuation quantities.

These equations are primarily used to describe turbulent flows and can be used with approximation based on knowledge of the properties of flow turbulence to give approximate time-averaged solutions to NavierStokes equations.

Compressible RANS equations, Favre-Average mean equations, are summarized below [4];

Continuity:

$$
\frac{\partial \bar{\rho}}{\partial t}+\frac{\partial}{\partial x_{i}}\left(\bar{\rho} \tilde{u}_{i}\right)=0
$$

Momentum:

$$
\frac{\partial}{\partial t}\left(\bar{\rho} \tilde{u}_{i}\right)+\frac{\partial}{\partial x_{j}}\left(\bar{\rho} \tilde{u}_{j} \tilde{u}_{i}\right)=-\frac{\partial P}{\partial x_{i}}+\frac{\partial}{\partial x_{j}}\left[\bar{t}_{j i}+\tau_{j i}\right]
$$

Energy:

$$
\begin{aligned}
\frac{\partial}{\partial t}(\bar{\rho} E) & +\frac{\partial}{\partial x_{j}}\left(\bar{\rho} \tilde{u}_{i} H\right)= \\
& =\frac{\partial}{\partial x_{j}}\left[-q_{L_{j}}-q_{T_{j}}+\overline{t_{j i} u_{i}^{\prime \prime}}-\overline{\rho u_{j}^{\prime \prime} \frac{1}{2} u_{i}^{\prime \prime} u_{i}^{\prime \prime}}\right]+ \\
& +\frac{\partial}{\partial x_{j}}\left[\tilde{u}_{i}\left(\bar{t}_{i j}+\tau_{i j}\right)\right]
\end{aligned}
$$

Turbulence kinetic energy:

$$
\begin{aligned}
\frac{\partial}{\partial t}(\bar{\rho} k) & +\frac{\partial}{\partial x_{j}}\left(\bar{\rho} \tilde{u}_{j} k\right)=\tau_{i j} \frac{\partial \tilde{u}_{i}}{\partial x_{j}}-\bar{\rho} \varepsilon+ \\
& +\frac{\partial}{\partial x_{j}}\left[\overline{t_{j i} u_{i}^{\prime \prime}}-\overline{\rho u_{j}^{\prime \prime}} \frac{1}{2} u_{i}^{\prime \prime} u_{i}^{\prime \prime}-\overline{p^{\prime} u_{j}^{\prime \prime}}\right]+ \\
& -\overline{u_{j}^{\prime \prime}} \frac{\partial P}{\partial x_{i}}+\overline{p^{\prime} \frac{\partial u_{i}^{\prime \prime}}{\partial x_{i}}}
\end{aligned}
$$

Perfect gas:

$$
P=\rho \bar{R} \tilde{T}
$$

The quantities $E$ and $H$ are the total energy and total enthalpy, and include the kinetic energy of the fluctuating turbulent field, hence:

$$
\begin{aligned}
& E=\tilde{e}+\frac{1}{2} \tilde{u}_{i} \tilde{u}_{i}+k \\
& H=\tilde{h}+\frac{1}{2} \tilde{u}_{i} \tilde{u}_{i}+k
\end{aligned}
$$

\section{II.1.1. Realizable $k-\varepsilon$}

Launder and Spalding [5] derived $k-\varepsilon$ model that is unarguably the most widely used and validate model employed for turbulent fluid dynamics to date. The extensive use of the model has highlighted both the capabilities and expresses of the model.

The formulation for Launder and Spalding's turbulence model consist of two transport equations, one equations to describe the kinetic energy of turbulence and a second related to the rate of turbulent dissipation [4], [6], [7], [8]. Turbulence Kinetic Energy:

$$
\begin{aligned}
\frac{\partial}{\partial t}(\bar{\rho} k)+\frac{\partial}{\partial x_{j}}\left(\bar{\rho} \tilde{u}_{j} k\right) & =+\frac{\partial}{\partial x_{j}}\left[\left(\mu+\frac{\mu_{t}}{\sigma_{k}}\right) \frac{\partial k}{\partial x_{j}}\right]+ \\
& +G_{k}+G_{b}+\rho \varepsilon-Y_{M}
\end{aligned}
$$

Specific Dissipation Rate:

$$
\begin{aligned}
\frac{\partial(\rho \varepsilon)}{\partial t}+\frac{\partial\left(\rho \tilde{u}_{j} \varepsilon\right)}{\partial x_{j}} & =\frac{\partial}{\partial x_{j}}\left[\left(\mu+\frac{\mu_{t}}{\sigma_{\epsilon}}\right) \frac{\partial \varepsilon}{\partial x_{j}}\right]+\rho C_{1} S \varepsilon+ \\
& -\rho C_{1} S \varepsilon-\rho C_{2} \frac{\varepsilon^{2}}{k+\sqrt{v \varepsilon}}+ \\
& +C_{1 \varepsilon} \frac{\varepsilon}{k} C_{3 \varepsilon} G_{b}
\end{aligned}
$$

where:

$$
\begin{gathered}
C_{1}=\max \left[0.43, \frac{\eta}{\eta+5}\right] \\
\eta=S \frac{k}{\varepsilon}
\end{gathered}
$$




$$
S=\sqrt{2 S_{i j} S_{i j}}
$$

In these equations, $G_{k}$ represents the function of generation of turbulence kinetic due to the mean velocity gradients. $G_{b}$ is the generation of turbulent kinetic energy due to buoyancy. $Y_{M}$ represents the contribution of the fluctuation dilatation in compressible turbulence to the overall dissipation rate. $C_{2}, C_{1 \varepsilon}$, and $C_{3 \varepsilon}$ are constant. $\sigma_{k}$ and $\sigma_{\epsilon}$ are the Prandtl numbers for $k$ and $\varepsilon$, respectively [7].

\section{II.1.2. SST $k-\omega$}

The shear-stress transport (SST) $k-\omega$ model including two equations was developed by Menter [4], [9], [10], [11], to effectively blend the robust and accurate formulation of the $k$ - $\omega$ model in the near wall region with the free-stream independence of the k- $\varepsilon$ model in the farfield [7]. Turbulence kinetic Energy:

$$
\begin{aligned}
\frac{\partial}{\partial t}(\bar{\rho} k) & +\frac{\partial}{\partial x_{j}}\left(\bar{\rho} \tilde{u}_{j} k\right)= \\
& =\frac{\partial}{\partial x_{j}}\left[\left(\mu+\frac{\mu_{t}}{\sigma_{k}}\right) \frac{\partial k}{\partial x_{j}}\right]+\tilde{G}_{k}-Y_{k}
\end{aligned}
$$

Specific Dissipation Rate:

$$
\begin{aligned}
\frac{\partial}{\partial t}(\bar{\rho} \omega)+\frac{\partial}{\partial x_{j}}\left(\bar{\rho} \tilde{u}_{j} \omega\right) & =\frac{\partial}{\partial x_{j}}\left[\left(\mu+\frac{\mu_{t}}{\sigma_{\omega}}\right) \frac{\partial \omega}{\partial x_{j}}\right]+ \\
& +G_{\omega}-Y_{\omega}+D_{\omega}
\end{aligned}
$$

In these equations, $\tilde{G}_{k}$ represents the generation of turbulence kinetic energy due to mean velocity gradient. $G_{\omega}$ represents the generation of $\omega . Y_{k}$ and $Y_{\omega}$ represent the $k$ and $\omega$ due to turbulence. $D_{\omega}$ represent the crossdiffusion term.

\section{II.1.3. Transition-SST}

The Transition-SST turbulence model is based on the coupling of the SST $k-\omega$ transport equations with two other transport equations, one for the intermittency and one for the transition onset criteria, in terms of momentum-thickness Reynolds number [7].

The transport equation for the intermittency $\gamma$ is defined as:

$$
\begin{aligned}
\frac{\partial(\rho \gamma)}{\partial t}+\frac{\partial\left(\rho U_{j} \gamma\right)}{\partial x_{j}} & =P_{\gamma 1}-E_{\gamma 1}+P_{\gamma 2}-E_{\gamma 2}+ \\
& +\frac{\partial}{\partial x_{j}}\left[\left(\mu+\frac{\mu_{t}}{\sigma_{\gamma}}\right) \frac{\partial \gamma}{\partial x_{j}}\right]
\end{aligned}
$$

The transition sources are defined as follows:

$$
\begin{gathered}
P_{\gamma 1}=C_{a 1} F_{\text {length }} \rho S\left[\gamma F_{\text {onset }}\right]^{c_{y 3}} \\
E_{\gamma 1}=C_{e 1} P_{\gamma 1} \gamma
\end{gathered}
$$

where $\mathrm{S}$ is the strain rate magnitude, $F_{\text {length }}$ is an empirical correlation that controls the length of the transition region, $C_{a 1}$ and $C_{e 1}$ hold the value of 2 and 1 , respectively.

The destruction/relaminarization sources are defined as follows:

$$
\begin{gathered}
P_{\gamma 2}=C_{a 2} \rho \Omega \gamma F_{\text {turb }} \\
E_{\gamma 2}=C_{e 2} P_{\gamma 2} \gamma
\end{gathered}
$$

where, $\Omega$ is the vorticity magnitude. The transition onset is controlled by the following functions:

$$
\begin{aligned}
& R e_{v}=\frac{\rho y^{2} S}{\mu} \\
& R_{T}=\frac{\rho k}{\mu \omega} \\
& F_{\text {onset } 1}=\frac{R e_{v}}{2.193 R e_{\theta c}} \\
& F_{\text {onset } 2}=\min \left(\max \left(F_{\text {onset } 1}, F_{\text {onset } 1}^{4}\right), 2.0\right) \\
& F_{\text {onset } 3}=\max \left(1-\left(\frac{R_{T}}{2.5}\right)^{3}, 0\right) \\
& F_{\text {onset }}=\max \left(F_{\text {onset } 2}-F_{\text {onset } 3}, 0\right) \\
& F_{\text {turb }}=e^{-\left(\frac{R_{T}}{4}\right)^{4}}
\end{aligned}
$$

$R e_{\theta c}$ is the critical Reynolds number where the intermittency first starts to increase in the boundary layer. This occurs upstream of the transition Reynolds number $R \tilde{e}_{\theta t}$ and the difference between the two must be obtained from an empirical correlation. Both the $F_{\text {length }}$ and $\operatorname{Re}_{\theta c}$ correlation are function of $R \tilde{e}_{\theta t}$. Where $C_{a 2}$, $C_{e 2}, C_{y 3}$ and $C_{S 1}$ are the constants for the intermittency equations [7]. The modification for separation-induced transition is:

$$
\gamma_{\text {sep }}=\min \left(C_{S 1} \max \left[\left(\frac{R e_{v}}{3.235 R e_{\theta c}}\right)-1,0\right] F_{\text {reattch }}, 2\right) F_{\theta l}(27)
$$




$$
\begin{gathered}
F_{\text {reattch }}=e^{-\left(\frac{R_{T}}{20}\right)^{4}} \\
\gamma_{\text {eff }}=\max \left(\gamma, \gamma_{\text {sep }}\right)
\end{gathered}
$$

The transition model interacts with SST turbulence model by modification of Eq. (10), as follows:

$$
\begin{aligned}
\frac{\partial}{\partial t}(\bar{\rho} k)+\frac{\partial}{\partial x_{j}}\left(\bar{\rho} \tilde{u}_{j} k\right) & =\frac{\partial}{\partial x_{j}}\left[\left(\mu+\frac{\mu_{t}}{\sigma_{k}}\right) \frac{\partial k}{\partial x_{j}}\right]+ \\
& +G_{k}^{*}-Y_{K}^{*}
\end{aligned}
$$

where:

$$
\begin{gathered}
G_{k}^{*}=\gamma_{e f f} \tilde{G}_{k} \\
Y_{k}^{*}=\min \left(\max \left(\gamma_{e f f}, 0.1\right), 1.0\right) Y_{k}
\end{gathered}
$$

\section{II.1.4. Reynolds-Stress (Stress-Omega)}

Launder initially derived the Reynolds-Stress model containing six equations and one length scale equation, after some modification on closure factor that developed by Wilcox[4], the Stress-omega follow as;

Reynolds-Stress Tensor:

$$
\begin{aligned}
\frac{\partial \bar{\rho} \tau_{i j}}{\partial t}+\frac{\left(\partial \bar{\rho} \tau_{i j} \tilde{u}_{k}\right)}{\partial x_{k}} & =-\bar{\rho} P_{i j}-\bar{\rho} \Pi_{i j}+\frac{2}{3} \beta^{*} \bar{\rho} \omega k \delta_{i j}+ \\
& +\frac{\partial}{\partial x_{k}}\left[\left(\bar{\mu}+\sigma^{*} \mu_{t}\right) \frac{\partial \tau_{i j}}{\partial x_{k}}\right]
\end{aligned}
$$

Specific Dissipation Rate:

$$
\begin{aligned}
\frac{\partial(\bar{\rho} \omega)}{\partial t}+\frac{\partial\left(\bar{\rho} \tilde{u}_{k} \omega\right)}{\partial x_{k}} & =\frac{\alpha \bar{\rho} \omega}{k} \tau_{i j} \frac{\partial \tilde{u}_{i}}{\partial x_{j}}-\beta \bar{\rho} \omega^{2}+ \\
& +\frac{\partial}{\partial x_{k}}\left[\left(\mu+\sigma \mu_{t}\right) \frac{\partial \omega}{\partial x_{k}}\right]+ \\
& +\sigma_{d} \frac{\bar{\rho}}{\omega} \frac{\partial k}{\partial x_{j}} \frac{\partial \omega}{\partial x_{j}}
\end{aligned}
$$

Production term is:

$$
P_{i j}=\tau_{i k} \frac{\partial \tilde{u}_{j}}{\partial x_{k}}+\tau_{j k} \frac{\partial \tilde{u}_{i}}{\partial x_{k}}
$$

The pressure-strain correlation is modeled via:

$$
\begin{aligned}
\Pi_{i j} & =\beta^{*} C_{1} \omega\left(\tau_{i j}+\frac{2}{3} k \delta_{i j}\right)-\hat{\alpha}\left(P_{i j}-\frac{2}{3} P \delta_{i j}\right)+ \\
& -\hat{\beta}\left(D_{i j}-\frac{2}{3} P \delta_{i j}\right)-\hat{\gamma} k\left(S_{i j}-\frac{1}{3} S_{k k} \delta_{i j}\right)
\end{aligned}
$$

Pressure dilatation is:

$$
D_{i j}=\tau_{i k} \frac{\partial \tilde{u}_{k}}{\partial x_{j}}+\tau_{j k} \frac{\partial \tilde{u}_{k}}{\partial x_{i}}
$$

Anisotropy tensor:

$$
b_{i j}=\frac{\overline{u_{i}^{\prime} u_{j}^{\prime}}-\frac{2}{3} k \delta_{i j}}{2 k}
$$

and other factors are closure factors referring to Wilcox [4].

\section{II.1.5. Spalart-Allmaras}

The compressible Spalart-Allmaras consisting of one equation was developed by Spalart and Allmaras [12].

Where, $G_{v}$ is the production of turbulent viscosity, and $Y_{v}$ is the destruction of turbulent viscosity that occurs in the near-wall region due to wall blocking and viscous damping. $\sigma_{\tilde{v}}$ and $C_{b 2}$ are the constant and $v$ is the molecular kinematic viscosity:

$$
\begin{aligned}
\frac{\partial(\rho \tilde{v})}{\partial t}+\frac{\partial\left(\rho \tilde{v} u_{j}\right)}{\partial x_{j}} & =\frac{1}{\sigma_{\tilde{v}}}\left[\frac{\partial}{\partial x_{j}}\left\{(\mu+\rho \tilde{v}) \frac{\partial \tilde{v}}{\partial x_{j}}\right\}\right]+ \\
& +\frac{1}{\sigma_{\tilde{v}}}\left[C_{b 2} \rho\left(\frac{\partial \tilde{v}}{\partial x_{j}}\right)\right]-Y_{v}+G_{v}
\end{aligned}
$$

\section{II.2. Noise Prediction Method}

In brief, the integration method of Ffowcs-Williams and Hawking is based on Lighthill's acoustic analogy. However, by some mathematical modification under the assumption of a limited source region, which is enclosed by a control surface (FW-H surface), the volume integral is avoided. Surface integrals over monopole and dipole sources remain.

Different from the Kirchhoff method, these sources ensue directly from the Navier-Stokes equations through Lighthill's analogy. Source outside the FW-H surface can be accounted by an additional volume integral over quadruple sources following form the Lighthill Tensor. However, when considering the same assumption as Kirchhoff's linear theory, the FW-H method equals the Kirchhoff method [13], [14].

\section{II.2.1. The Ffowcs-William and Hawkings Model}

The Ffowcs-William and Hawkings (FW-H) equation is essentially an inhomogeneous wave equation that can be derived by manipulating the continuity equation and the Navier-Stokes equations. The FW-H equations can be written as [7], [14]: 


$$
\begin{aligned}
\frac{1}{C_{0}^{2}} \frac{\partial^{2} p^{\prime}}{\partial t^{2}} & -\nabla^{2} p^{\prime}=\frac{\partial^{2}}{\partial x_{i} \partial x_{j}}\left\{T_{i j} H(f)\right\}+ \\
& -\frac{\partial}{\partial x_{i}}\left\{\left[P_{i j} n_{j}+\rho u_{i}\left(u_{n}-v_{n}\right)\right] \delta(f)\right\}+ \\
+ & \frac{\partial}{\partial t}\left\{\left[\rho_{0} v_{n}+\rho\left(u_{n}-v_{n}\right)\right] \delta(f)\right\}
\end{aligned}
$$

$f=0$ denotes a mathematical surface introduce to "embed" the exterior flow problem $(f>0)$ in an unbounded space, which facilitates the use of generalized function theory and the free-space Green function to obtain the solution. The surface $(f=0)$ corresponds to the source (emission) surface, and can be made coincident with a body (impermeable) surface or a permeable surface off the body surface. $n_{j}$ is the unit normal vector pointing toward the exterior region $(f>0), C_{0}$ is the local sound speed, and $T_{i j}$ is the Lighthill stress tensor, define as:

$$
T_{i j}=\rho u_{i} u_{j}+P_{i j}-C_{0}^{2}\left(\rho-\rho_{0}\right) \delta_{i j}
$$

$P_{i j}$ is the compressive stress tensor. For a Stokesian fluid, this is given by:

$$
P_{i j}=p \delta_{i j}-\mu\left[\frac{\partial u_{i}}{\partial x_{j}}+\frac{\partial u_{j}}{\partial x_{i}}-\frac{2}{3} \frac{\partial u_{k}}{\partial x_{k}} \delta_{i j}\right]
$$

The wave equation Eq. (40) can be integrated analytically under the assumptions of the free-space flow and the absence of obstacles between the sound sources and the receivers. The complete solution consists of surface integrals and volume integrals. The surface integrals represent the contributions from monopole and dipole acoustic sources and partially from quadruple sources, whereas the volume integrals represent quadruple (volume) sources in the region outside the source surface [7]. It actually states that the contribution of the volume integrals becomes small when the flow is low subsonic and the source surface encloses the source region. The volume integrals are dropped. Thus, we have:

$$
p^{\prime}(\vec{x}, t)=p_{T}^{\prime}(\vec{x}, t)+p_{L}^{\prime}(\vec{x}, t)
$$

where:

$$
\begin{aligned}
& 4 \pi p_{T}^{\prime}(\vec{x}, t)=\int_{f=0}\left[\frac{\rho_{0}\left(\dot{U}_{n}+U_{\dot{n}}\right)}{r\left(1-M_{r}\right)^{2}}\right] d S+ \\
& +\int_{f=0}\left[\frac{\rho_{0} U_{n}\left\{r \dot{M}_{r}+C_{0}\left(M_{r}-M^{2}\right)\right\}}{r^{2}\left(1-M_{r}\right)^{3}}\right] d S
\end{aligned}
$$

$$
\begin{aligned}
4 \pi p_{L}^{\prime}(\vec{x}, t) & =\frac{1}{C_{0}} \int_{f=0}\left[\frac{\dot{L}_{r}}{r\left(1-M_{r}\right)^{2}}\right] d S+ \\
& +\int_{f=0}\left[\frac{L_{r}-L_{M}}{r^{2}\left(1-M_{r}\right)^{2}}\right] d S+ \\
& +\frac{1}{C_{0}} \int_{f=0}\left[\frac{L_{r}\left\{r \dot{M}_{r}+C_{0}\left(M_{r}-M^{2}\right)\right\}}{r^{2}\left(1-M_{r}\right)^{3}}\right] d S
\end{aligned}
$$

where:

$$
\begin{gathered}
U_{i}=v_{i}+\frac{\rho}{\rho_{0}}\left(u_{i}-v_{i}\right) \\
L_{i}=P_{i j} n_{j}+\rho u_{i}\left(u_{n}-v_{n}\right)
\end{gathered}
$$

when the integration surface coincides with an impenetrable wall, the two terms on the right in Eq. (40), $p_{T}^{\prime}(\vec{x}, t)$ and $p_{L}^{\prime}(\vec{x}, t)$, are often referred to as thickness (Monopole) and loading (Dipole) terms [15], respectively, in light of their physical meaning.

The square brackets in Eq. (44) and Eq. (45) denote that the kernels of the integrals are computed at the corresponding retarded time, $\tau$ defined as follows, given the receiver time, $t$, and the distance to the receiver, $r$ :

$$
\tau=t-\frac{r}{C_{0}}
$$

The various subscripted quantities appearing in Eq. (44) and Eq. (45) are the inner products of a vector and a unit vector implied by the subscript. For instance, $L_{r}=\vec{L} \cdot \vec{r}=L_{i} r_{i}$ and $U_{n}=\vec{U} \cdot \vec{n}=U_{i} n_{i}$, where $\vec{r}$ and $\vec{n}$ denote the unit vectors in the radiation and wall-normal directions, respectively. The Mach number vector $M_{i}$ in Eq. (41) and Eq. (42) relates to the motion of the integration surface. The $L_{i}$ quantity is scalar product $L_{i} M_{i}$. The dot over a variable denotes source-time differentiation of that variable. To analyze the data, research considered Fast-Fourier Transform (FFT) to plot the semi-logarithmic Sound Pressure Level (SPL) vs. Frequency and calculates with Eq. (49):

$$
\operatorname{SPL}\left(f_{n}\right)=10 \log \left(\frac{p^{\prime 2}\left(f_{n}\right)}{p_{\text {ref }}^{2}}\right)
$$

where, $p^{\prime}\left(f_{n}\right)$ is the power spectral density of the pressure fluctuation and $p_{\text {ref }}$ is the reference acoustic pressure. 
Brooks specified equation to account the equal contribution of the two sides of airfoil for the total Spectrum [3]. Eq. (50) is For the pressure side and suction side, $i=p, s$ :

$$
S P L_{\text {Total }}=10 \log \left(10^{\frac{S P L_{S}}{10}}+10^{\frac{S P L p}{10}}\right)
$$

Tian et al. [16] introduced a near-field acoustic emission (AE) beamforming method to estimate the $\mathrm{AE}$ source location, but it allocates to the solid mechanic. Aeroacoustics relates to fluid mechanics, therefore this paper seeks to define new method to analyze the critical sources and fluctuated points calling Acoustic Localization Analysis (ALA).

For this reason, with the aid of numerical analysis, the trapezoidal rule of integral is used to generate AverageDependable SPL for acoustical analysis of specifically located receivers.

This parameter is defined including location, range of frequency, and corresponding SPL of each receivers to help finding critical location of fluctuations and consequently noise production, which is more explained in section VI.

\section{CFD Verification and Validation}

All CFD computations were performed under the commercial CFD code. Using structural O-Type mesh, the chord length based on the $22.86(\mathrm{~cm})$. The computations use Finite Volume Method (FVM), second order and second implicit solver for unsteady flow. The turbulence intensity has a value of 1 percent.

The verification also is divided into the two sections. In Aerodynamic section, it compares both drag coefficient and pressure coefficient with experimental data.

In Aero-acoustic section, it compares semilogarithmic Sound Pressure Level vs. Frequency with Brooks' data [3]. Fig. 5 and Fig. 6 show the qualities of mesh and its schematic of CFD domain.

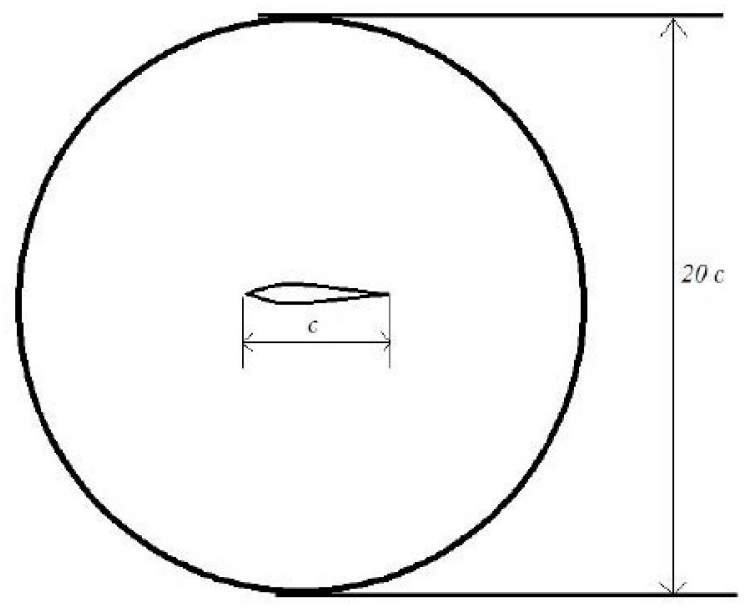

Fig. 5. Schematic of CFD domain (c is chord length)

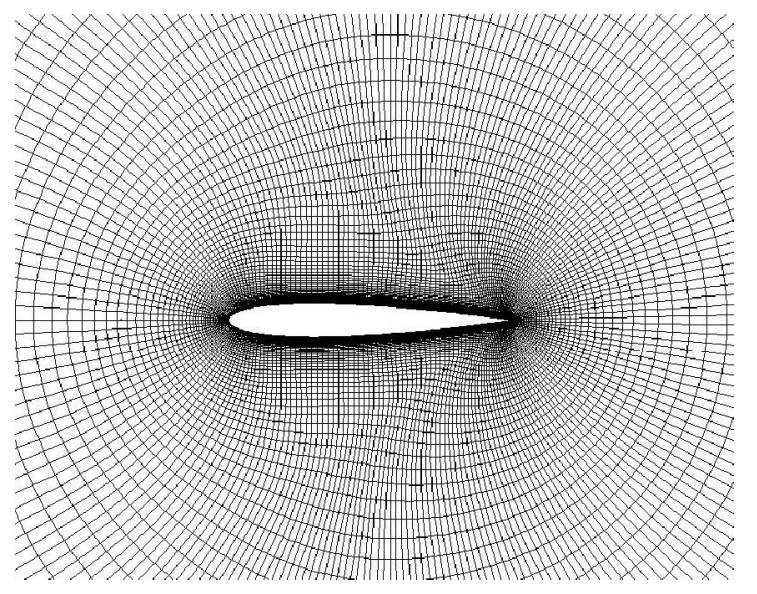

Fig. 6. Close-up view of the CFD meshes for the NACA 0012 airfoil

\section{III.1. Grid Study}

Table II and Table III show the flow specification and grid study result, respectively.

The Drag coefficient is compared with McCrosley's data [17]. In Fig. 7, the pressure coefficient is compared with Ladson's data [18], [19] for grid independent study of three meshes. 0.55 .

In addition, Fig. 8 pictured Mach number contour of

TABLE II

FLOW SPECIFICATION

Mach 0.55

Reynolds $\quad 3000000$

Chord $(\mathrm{cm}) \quad 22.86$

AoA Zero

Turbulence Model Transition-SST

\begin{tabular}{cc} 
Turbulence Intensity & $1 \%$ \\
\hline \hline
\end{tabular}

TABLE III

GRID STUDY

\begin{tabular}{ccccc}
\hline \hline Mesh & \# Cells & Max of y+ & $C_{D}$ & Error \% \\
\hline 1 & 16875 & 11.3 & 0.010011 & 5.4 \\
2 & 35970 & 9.4 & 0.009625 & 1.3 \\
3 & 71940 & 9.2 & 0.009656 & 1.6 \\
\hline \hline
\end{tabular}

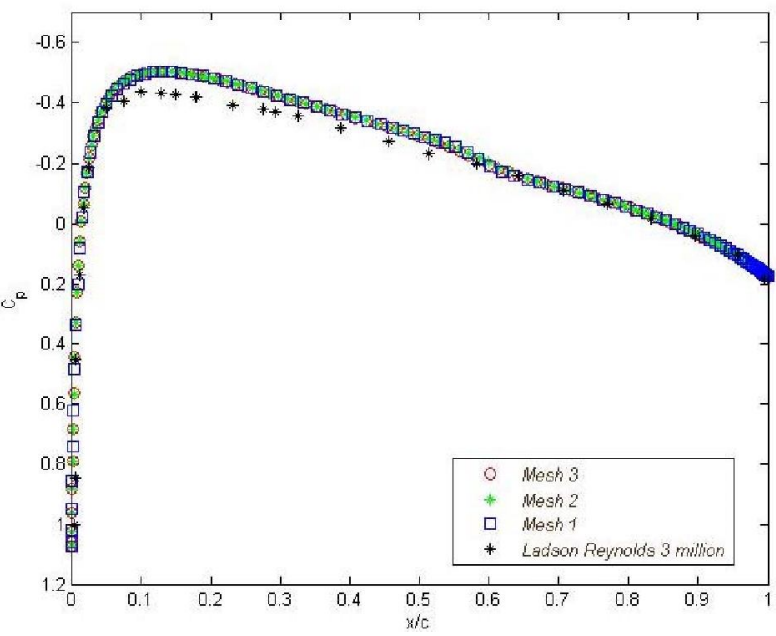

Fig. 7. Pressure coefficient for 3 meshes at zero angle of attack 


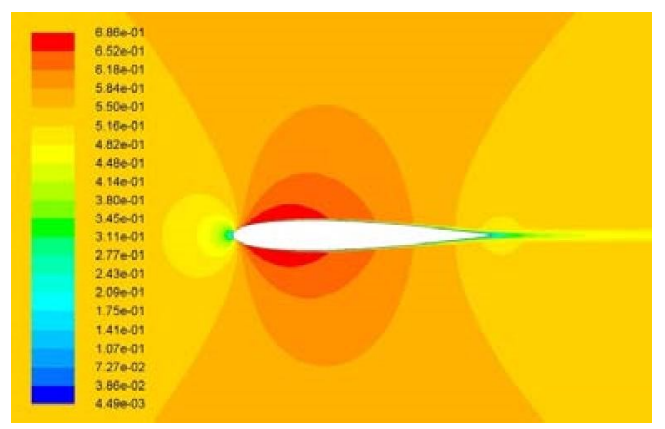

Fig. 8. Contour of Mach equals to 0.55 for Mesh \#1

\section{Aerodynamic Results}

After that CFD validation at the Mach number of 0.55 , research focused on a Mach number of 0.209 including velocity of 71.3 to compute the acoustic data of defining receivers. Fig. 9 shows the pressure coefficient, Fig. 10 and Fig. 11 show the contour of Mach number and turbulence intensity at the speed of Mach 0.209 .

\section{Acoustic Results}

This section is allocated to the predicted noise spectra of the NACA 0012 airfoil at a speed of 71.3 meter per second for five turbulence model. The Reynolds number corresponds to $1.108761 \times 10^{6}$. Fig. 12 to Fig. 16 are for realizable $k-\varepsilon$, SST $k-\omega$, Transition-SST, Reynolds Stress, and Spalart-Allmaras turbulence models, consecutively.

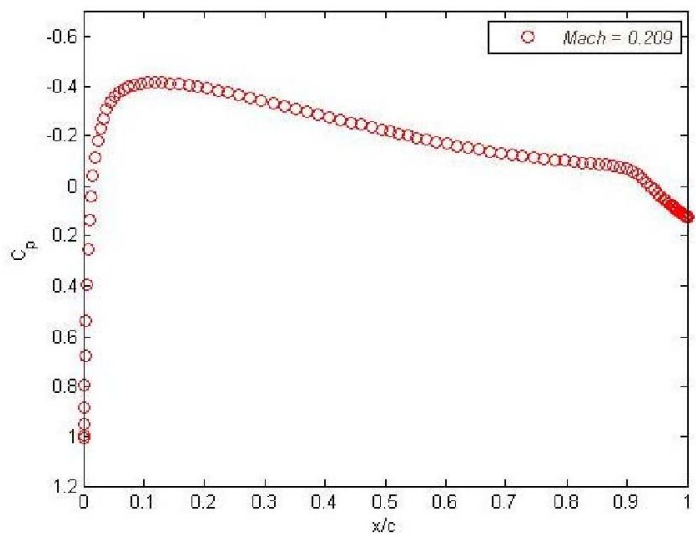

Fig. 9. Pressure coefficient for Reynolds $=1.1 \times 10^{6}$

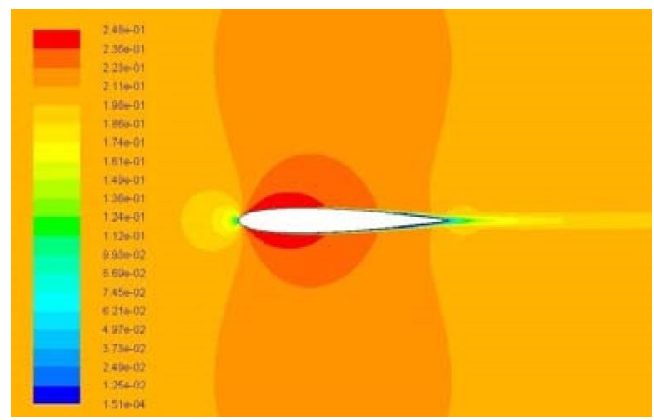

Fig. 10. Contour of Mach equals to 0.209

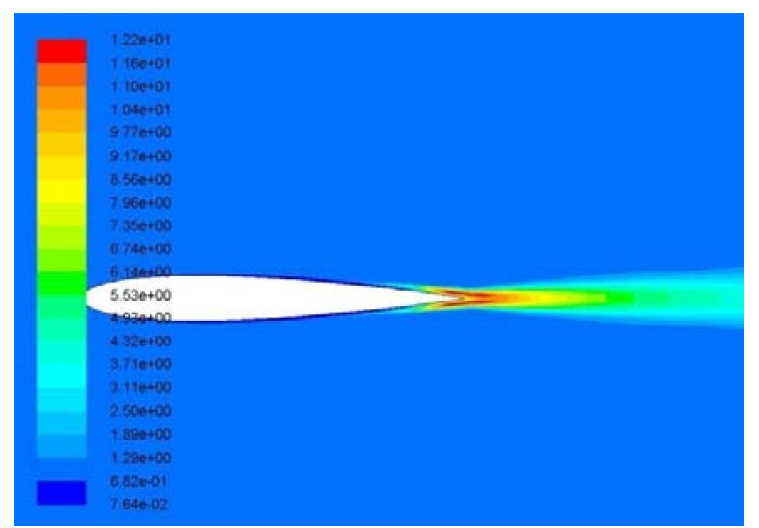

Fig. 11. Contour of Turbulence Intensity for Transition-SST model

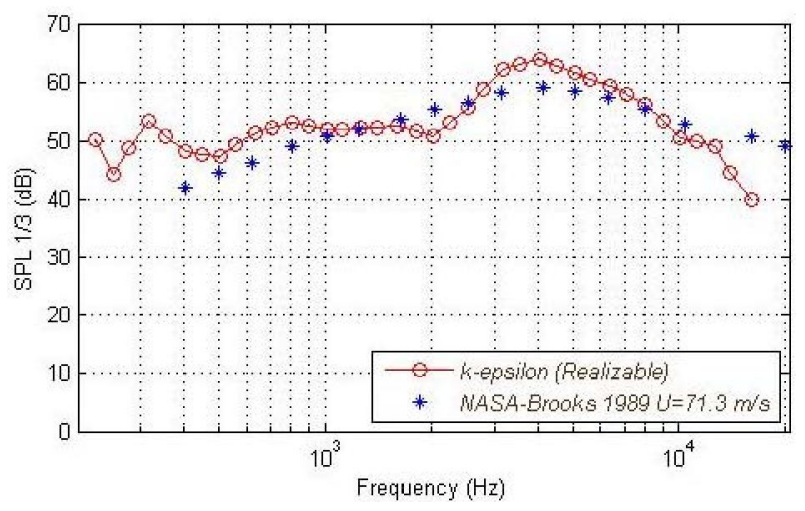

Fig. 12. Realizable $k-\varepsilon$

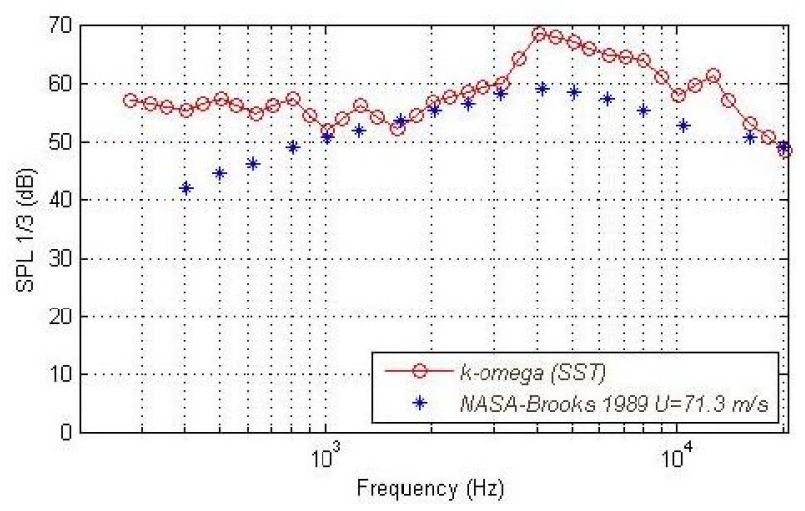

Fig. 13. SST $k-\omega$

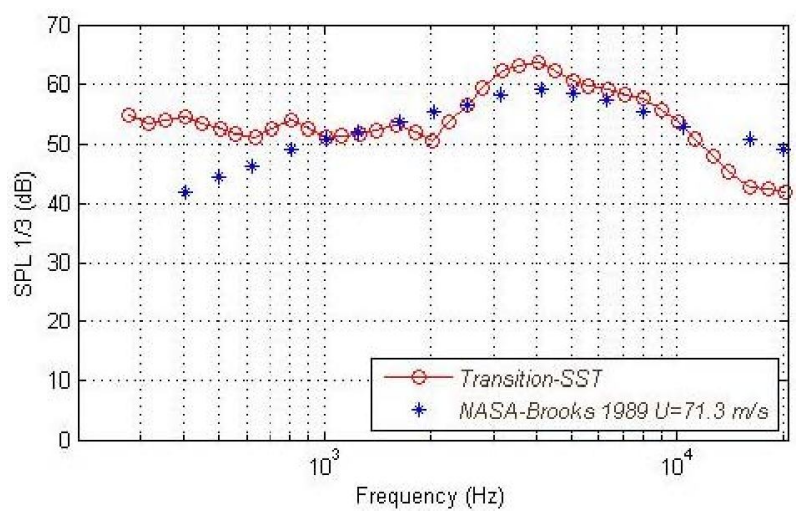

Fig. 14. Transition-SST 


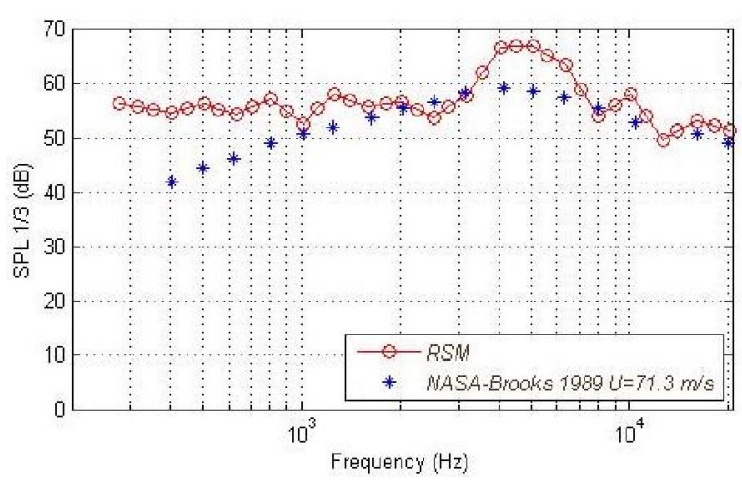

Fig. 15. Reynolds Stress

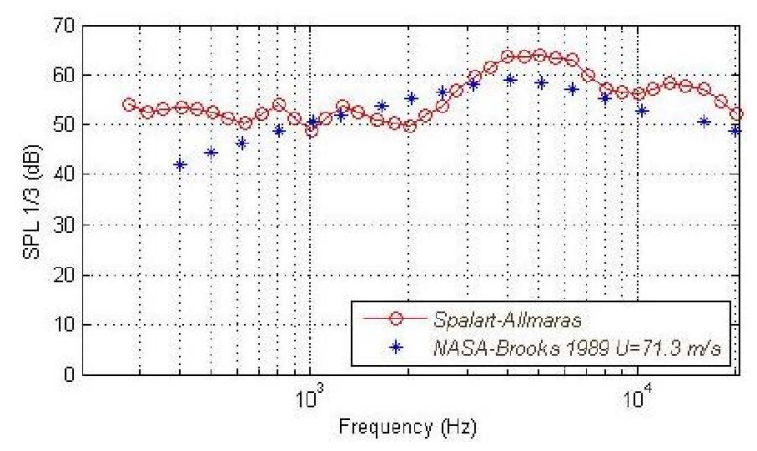

Fig. 16. Spalart-Allmaras

\section{V.1. Acoustic Errors}

There are three criteria to analyze the accuracy of acoustic curves that can be calculated as Eq. (51) to Eq. (53), Where, PPE is Predicted Peak Error, PFPE is Predicted Frequency of Peak Error, and TRE is Total Residual Error:

$$
\begin{gathered}
P P E=\frac{\left|P e a k_{\text {expr }}-P e a k_{N S}\right|}{P e a k_{\text {expr }}} \times 100 \\
P F P E=\frac{\left|F_{\text {expr }}-F_{N S}\right|}{F_{\text {expr }}} \times 100 \\
T R E=\frac{\left|\sum S P L_{\text {expr }}-\sum S P L_{N S}\right|}{\sum S P L_{\text {expr }}} \times 100
\end{gathered}
$$

\begin{tabular}{|c|c|c|c|c|}
\hline & Turbulence Models & $\begin{array}{l}\text { PPE } \\
(\%)\end{array}$ & $\begin{array}{c}\text { PFPE } \\
(\%)\end{array}$ & TRE $(\%)$ \\
\hline 1 & k- $\varepsilon$ (Realizable) & 8.1 & 1.9 & 6.1 \\
\hline 2 & $\mathrm{k}-\omega(\mathrm{SST})$ & 15.9 & 1.9 & 11.2 \\
\hline 3 & Transition-SST & 7.5 & 1.9 & 7.7 \\
\hline 4 & Reynolds Stress & 13.1 & 22.9 & 10.1 \\
\hline 5 & Spalart-Allmaras & 8.2 & 22.9 & 7.6 \\
\hline
\end{tabular}

TABLE IV ACOUSTIC ERRORS

In order to do further discussion about the acoustic errors, this paper used a set of criteria for the errors of numerical simulation below the $10 \%$.
It can be observed that realizable $k$ - $\varepsilon$, Transition-SST, and Spalart-Allmaras have a fair agreement with the experimental data for the predictive amount of peak. For the predicted frequency of peak, the realizable $k-\varepsilon$, SST $k-\omega$, and Transition-SST have reasonable errors under $2 \%$. For the total residual error, the paper considered the accuracy of each of numerical simulation with the experimental data to determine which ranges of frequency are more accurate to do further research in near future.

Fig. 12 shows that realizable $k-\varepsilon$ model has good prediction in low frequency in contrast to other turbulence models. On the contrary, the accuracy of this model deteriorates at a frequency above $15 \mathrm{KHz}$. Both Fig. 15 and Fig. 16, Reynolds-Stress and SpalartAllmaras Turbulence models, represent the fair data but their predicted frequency of peak have substantial difference with the experimental data. Since realizable $k$ $\varepsilon$ and Transition-SST have more accurate data than other turbulence models and in retrospect to the shorter run time of realizable $k-\varepsilon$ models, research focuses on this model to do Acoustic Localization Analysis (ALA).

\section{Acoustic Localization Analysis}

The focus of this section issued some analysis of the locations of 19 receivers due to the homogenous distribution in semi-circle circumference around the airfoil, Fig. 17, and a far-field linear distribution with constant y components, Fig. 18, respectively.

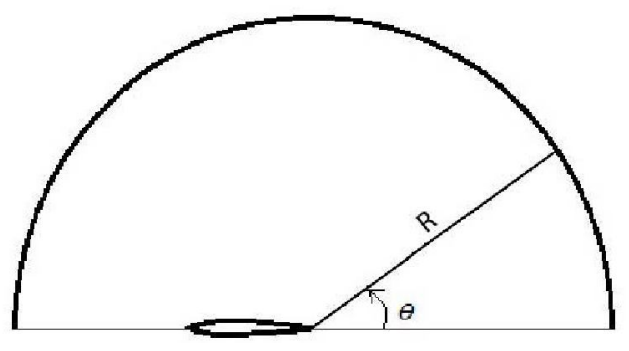

Fig. 17. Schematic of semi-circle circumference

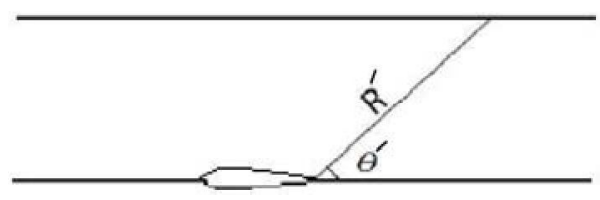

Fig. 18. Schematic of far-field linear distribution

In Fig. 17, the center of rotation corresponds to trailing edge and the equations of 19 located receivers in structural grid follow as:

$$
\begin{gathered}
x=c+R \cos (\theta) \\
y=R \sin (\theta) \\
R=\text { const }
\end{gathered}
$$


For Fig. 18, the center of rotation also corresponds to the trailing edge and the equations of 19 located receivers are:

$$
\begin{gathered}
x^{\prime}=c+R^{\prime} \cos \left(\theta^{\prime}\right) \\
y^{\prime}=\text { const } \\
R^{\prime}=\frac{y^{\prime}}{\sin \left(\theta^{\prime}\right)}
\end{gathered}
$$

The correlation between far-field linear and semicircle distribution is:

$$
\begin{gathered}
x^{\prime}=x \\
\tan \left(\theta^{\prime}\right)=\frac{1}{\cos (\theta)}
\end{gathered}
$$

In addition, for Fig. 19, the equations of 10 receivers follow as:

$$
\begin{gathered}
i=0,1,2, \ldots, 10 \\
x^{\prime \prime}=i \frac{c}{10} \\
y^{\prime \prime}=\text { const } \\
y=\text { const } \\
\end{gathered}
$$

Fig. 19. Schematic of linear distribution

The retard time, Eq. (48), is important to analyze the critical peak because of correlation between location of noise sources and receivers in unsteady flow.

In fact, there are several factors that have close relation to the sound wave interaction, such as local sound speed, frequency, location and the vorticity magnitude of local pressure fluctuation close to the receiver. Fig. 20 shows the slice of fluctuation for quadruple sources of random distribution, which is simulated to enlighten the retard time fact and sound sources interaction.

To compare all receivers and their recorded fluctuations of pressure, a parameter is defined to plot a curve relating to the locations, frequencies and SPLs of each receiver that we call it Average-Dependable SPL. It is basically the Numerical Integral of SPL vs. Frequency curve and its unit follows as (dBSPL.Hz).

In Fig. 21, it can be also observed that there are some explanation to discuss in each curve, which needs to follow up. The turbulence flow passed over TE, including wakes, within the range of zero to 70 degree has similar behaviors in each radius; the Leading-Edge (LE) and TBL-TE noise within the range of 70 to 110 degree are dominated because of the several sound wave interactions (peaks) such as, Monopole, Dipole, and Quadruple. From the angle of 110 to 180 , with the increase in distance, there is chaos behavior respect to the retard time fact. Since these locations are approximately closer to LE than TBL-TE, the LE noise is more conspicuously dominated. Brooks et al explained that for the larger airfoils, the TE contribution dominates the noise field. As the chord length decreases, the LE noise peaks increase to become readily identifiable in the correlation and more than TE noise [3].

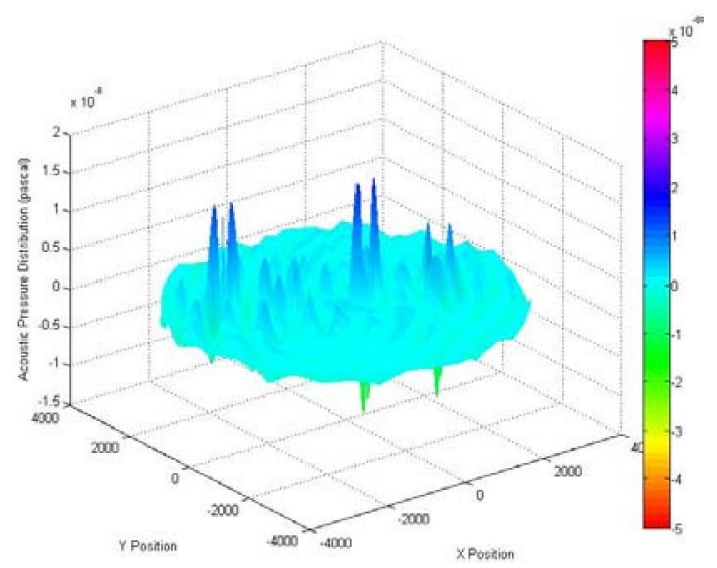

Fig. 20. The impacts of retard time on captured SPL of receiver

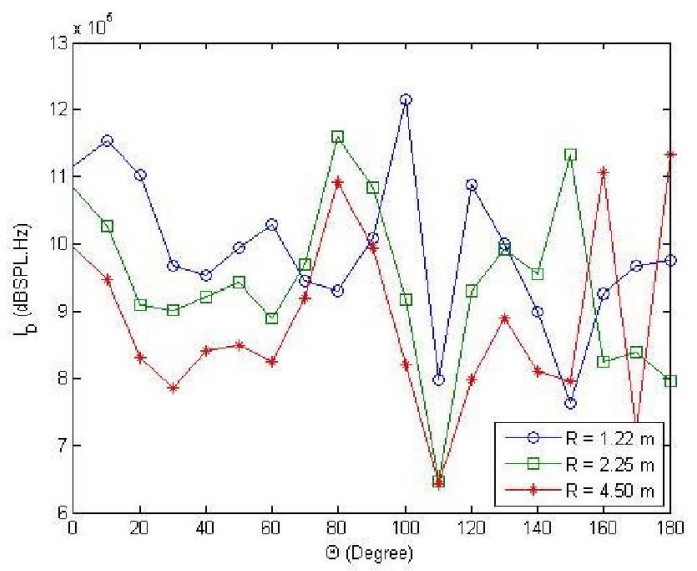

Fig. 21. Average-Dependable SPL of semi-circle distribution

Fig. 22 shows that some of peaks are close to LE. Increasing in distance from airfoil indicates that flow passed over the TE including the locations of TBL, wakes, and maximum of $T i$ of flow for realizable $k-\varepsilon$ model, Fig. 23, generates TE noise. 